\title{
Validation of gyrokinetic simulations with measurements of electron temperature fluctuations and density-temperature phase angles on ASDEX Upgrade
}

\author{
S.J. Freethy ${ }^{1,2}$, T. Görler ${ }^{1}$, A.J. Creely $^{2}$, G.D. Conway ${ }^{1}$, T. Happel ${ }^{1}$,C. \\ Koenen $^{3}$ P. Hennequin ${ }^{4}$, A.E. White ${ }^{2}$, the ASDEX Upgrade Team, ${ }^{2}$, \\ ${ }^{1}$ Max Plank Institute for Plasma Physics, 85748 Garching, Germany \\ ${ }^{2}$ Plasma Science and Fusion Center, Massachusetts Institute of Technology, Cambridge, MA 02139, USA \\ ${ }^{3}$ Lehrstuhl für Hochfrequenztechnik, Technische Universität München, Arcisstr. 21, 80333 München and \\ ${ }^{4}$ Laboratoire de Physique des Plasmas, Ecole Polytechnique, 91128, Palaiseau cedex, France
}

(Dated: February 23, 2018)

\begin{abstract}
Measurements of turbulent electron temperature fluctuation amplitudes, $\delta T_{e \perp} / T_{e}$, frequency spectra and radial correlation lengths, $L_{r}\left(T_{e \perp}\right)$, have been performed at ASDEX Upgrade using a newly upgraded Correlation ECE diagnostic in the range of scales $k_{\perp}<1.4 \mathrm{~cm}^{-1}, k_{r}<3.5 \mathrm{~cm}^{-1}$ $\left(k_{\perp} \rho_{s}<0.28\right.$ and $\left.k_{r} \rho_{s}<0.7\right)$. The phase angle between turbulent temperature and density fluctuations, $\alpha_{n T}$, has also been measured by using an ECE radiometer coupled to a reflectometer along the same line of sight. These quantities are used simultaneously to constrain a set of ion-scale nonlinear gyrokinetic turbulence simulations of the outer core $\left(\rho_{\text {tor }}=0.75\right)$ of a low density, electron heated L-mode plasma, performed using the gyrokinetic simulation code, GENE. The ion and electron temperature gradients were scanned within uncertainties. It is found that GK simulations are able to match simultaneously the electron and ion heat flux at this radius within the experimental uncertainties. The simulations were performed based on a reference discharge for which $\delta T_{e \perp} / T_{e}$ measurements were available, and $L_{r}\left(T_{e \perp}\right)$ and $\alpha_{n T}$ were then predicted using synthetic diagnostics prior to measurements in a repeat discharge. While temperature fluctuation amplitudes are overestimated by $>50 \%$ for all simulations within the sensitivity scans performed, good quantitative agreement is found for $L_{r}\left(T_{e \perp}\right)$ and $\alpha_{n T}$. A validation metric is used to quantify the level of agreement of individual simulations with experimental measurements, and the best agreement is found close to the experimental gradient values.
\end{abstract}

\section{INTRODUCTION}

Understanding the turbulent driven heat flux in a tokamak remains one of the key goals of fusion research. Anomalous transport up to two orders of magnitude above what one would expect from neoclassical theory is observed and this is now understood to be caused by turbulent fluctuations in the plasma density, temperature and potential, originating from drift-wave like instabilities [1,2]. The most complete, tractable models of this turbulence are currently given by numerical approaches to the gyrokinetic (GK) equations [3]. Much progress has been made over the past few decades in the development of gyrokinetic approaches and in validating non-linear gyrokinetic codes against experiment. In some cases, validation studies performed with these codes are able to match the experimental heat flux for both ions and electrons within experimental error bars [4-9]. However, there remain many examples where this is not the case, with the simulations unable to match the ion heat flu, $Q_{i}[10,11]$ or the electron heat flux, $Q_{e}[12-$ 15]. In the cases where ion scale simulations are unable to match the electron heat flux, it has been suggested that electron scale turbulence may be playing a role, either directly $[16,17]$ or through non-linear multi-scale coupling of ion and electron turbulence [18-20]. Since this latter case requires simulations which are presently almost prohibitively computationally expensive, it is of a very high importance to identify the region of parameter space where single scale simulations suffice.
For the situations where the models are able to reproduce the experimental heat fluxes, it is important to understand whether they do this with a physically realistic model. This can only be done by quantitatively comparing the simulation to one or more experimentally measured properties of the turbulence, known in the community as a validation study [21]. At ASDEX Upgrade (AUG), in order to probe the low wavenumber $(k)$ temperature fluctuations contributing to the electron heat flux channel and to compliment the existing turbulence diagnostics used for GK validation [22], a Correlation Electron Cyclotron Emission (CECE) diagnostic has been installed $[23,24]$. The principles of this diagnostic are briefly outlined in Section III. Using a unique channel comb, this diagnostic can measure high radial resolution $\delta T_{e \perp} / T_{e}$ and radial correlation length $L_{r}\left(T_{e \perp}\right)$ profiles. The CECE diagnostic also shares a line of sight to the plasma with a number of reflectometers [25-27], which are sensitive to the fluctuating density component of the turbulence. This allows simultaneous, co-located measurements of density and temperature fluctuations and their corresponding cross-phase angle $\alpha_{n, T}$.

Measurements of $\delta T_{e \perp} / T_{e}$, frequency spectra, $L_{r}\left(T_{e \perp}\right)$ and $L_{r}\left(T_{e \perp}\right)$, have been performed in an ASDEX Upgrade (AUG) L-mode plasma. These quantities are used simultaneously to constrain a set of ion-scale non-linear gyrokinetic turbulence simulations of the outer core of a low density, electron heated L-mode plasma, performed using the gyrokinetic simulation code, GENE. The gradient scale lengths of the $T_{i}$ and the $T_{e}$ profile were varied 
within uncertainties to assess the sensitivity of the simulations to these parameters. It is found that the GK simulations are able to match simultaneously the $Q_{i}$ and $Q_{e}$ at this radius within the experimental uncertainties. While temperature fluctuation amplitudes are overestimated by $>50 \%$ for all simulations within the sensitivity scans performed, good quantitative agreement is found for $L_{r}\left(T_{e \perp}\right)$ and $\alpha_{n T}$. A validation metric is used to quantify the level of agreement of individual simulations with experimental measurements, and the best agreement is found close to the experimental gradient values.

The paper is organised as follows: Section II contains a description of the plasma discharges used for the validation study, Section III details the experimental techniques used to measure the fluctuating quantities which are subsequently compared to the simulations, Section IV describes the simulations of the plasmas and the synthetic diagnostics used to make the quantitative comparisons, Section IVE describes an attempt to use a validation metric to quantitatively compare the simulations within the sensitivity scans and Section V is a summary of the observations and presents some concluding remarks.

\section{L-MODE PLASMA USED FOR GK VALIDATION}

In this study measurements of the temperature fluctuation amplitude, $\delta T_{e \perp} / T_{e}$, the radial correlation length, $L_{r}\left(T_{e \perp}\right)$, and the cross-phase angle between temperature and density fluctuations, $\alpha_{n T}$ are used. These measurements were taken during two discharges in the ASDEX Upgrade (AUG) experiment, a reference discharge (\#33585) in which only $\delta T_{e \perp} / T_{e}$ is available from the pilot CECE diagnostic [23], and a repeat discharge (\#34626) where all the aforementioned fluctuation quantities are available from a newly upgraded CECE diagnostic [24].

The gyrokinetic simulations presented in this paper are all based on the reference discharge. This discharge is a low density, predominantly electron heated L-mode plasma, with magnetic field on axis $B=2.5 \mathrm{~T}$, plasma current $I_{p}=1.0 \mathrm{MA}$. The applied heating was Ohmic and Electron Cyclotron Resonance Heating (ECRH) with a power $P_{\mathrm{ECRH}}=0.7 \mathrm{MW}$. The reference L-mode plasma is stationary for a period of $2 \mathrm{~s}$ and it is over this time that the profile measurements are averaged. The repeat discharge is stationary for $3.3 \mathrm{~s}$ allowing a longer period for averaging the fluctuation measurements. For the reference discharge, Figure 1 shows as a function of the normalised toroidal flux radius, $\rho_{\text {tor }}$, the electron temperature measured with a profile radiometer [28], electron density measured with Thomson Scattering $[29,30]$ and ion temperature and toroidal rotation profile measured using a charge exchange recombination spectroscopy (CXRS) system combined with periodic neutral beam blips [31]. The effective plasma charge $z_{\text {eff }}$, is calculated from the Bremsstrahlung radiation measured by the CXRS diagnostic and is $1.6+/-0.2$ for the reference case. The uncertainty on this value is significantly higher for the repeat discharge, but consistent with the reference. Characteristic uncertainties for these parameters can be found in the respective publications, however at the radial location of interest the uncertainties $\sigma_{T_{e}} \simeq 15 \%$, $\sigma_{n_{e}} \simeq 10 \%, \sigma_{T_{i}} \simeq 20 \%$ and $\sigma_{v_{\phi}} \simeq 50 \%$. The profile fits to these data are also shown as solid lines. Panel b) of Figure 1 shows the profiles of the normalised scale lengths of these quantities and the dashed line indicates the radius for which the gyrokinetic simulations were performed. Thomson and ECE profile data are not available over the radial range $\rho_{\text {tor }}=0-0.2$, however this is not thought to affect the profile fits at the simulation radius.. In this paper we define the inverse gradient scale length of a quantity $X, 1 / L_{X}$, as $-d / d \rho_{\text {tor }}(\ln X)$. This is sometimes referred to in the literature as $\omega_{X}$. Uncertainties in the scale lengths come from a combination of the inherent scatter in the data and the method used to calculate the profile fits. It was estimated using a Monte Carlo approach that the normalized gradient scale length uncertainties were $20 \%$ for $1 / L_{T_{e}}, 30 \%$ for $1 / L_{T_{i}}$, and $30 \%$ for $1 / L_{n_{e}}$ at $\rho_{t o r}=0.75$. The profiles and scale lengths in the repeat discharge match the reference discharge within uncertainties from $\rho_{\text {tor }}=0.25-1.00$. Further, $\delta T_{e \perp} / T_{e}$ is also in agreement within uncertainties.
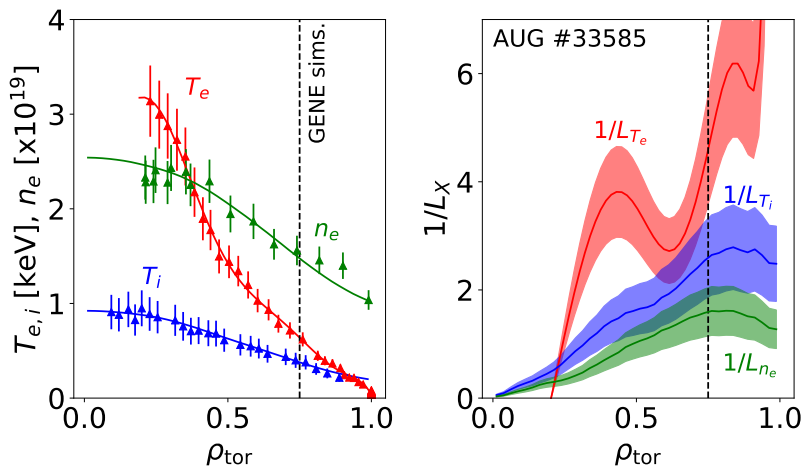

FIG. 1. Measured kinetic profiles a) and scale lengths b) for the reference discharge AUG 33585.

Power balance transport analysis for the reference discharge considered in this study was performed with the transport code TRANSP [32]. The input uncertainties were propagated through the governing transport equations solved by TRANSP to obtain uncertainties on the output heat fluxes. This calculation leads to conductive heat flux values of $Q_{i}=(0.33 \pm 0.10) \mathrm{MW}$ and $Q_{e}=(0.8 \pm 0.13) \mathrm{MW}$ to an uncertainty of $26 \%$ for $Q_{i}$ and $14 \%$ for $Q_{e}$ and at $\rho_{t o r}=0.75$. 


\section{TURBULENCE MEASUREMENT TECHNIQUES}

\section{A. Correlation ECE measurements of $\delta T_{e \perp}$ fluctuations}

Under typical tokamak conditions, core turbulent electron temperature fluctuations are sufficiently broadband $(\sim 0.5 \mathrm{MHz})$ and low-amplitude $(\sim 1 \%)$ that a conventional radiometer is fundamentally unable to detect them [33]. However, correlation techniques can further reduce the intrinsic noise and extract the small, wideband turbulent fluctuation signals [33-35]. For a review of CECE types and techniques see [36]. The AUG CECE diagnostic [23] is of the spectral decorrelation type, meaning it is an ECE radiometer with channels sensitive to ECE radiation in distinct, non-overlapping narrow frequency bands, which are spaced sufficiently closely that neighbouring pairs are sensitive to the same turbulent temperature fluctuations. Correlation analysis between channel pairs then allows the uncorrelated thermal noise of each channel to be significantly reduced, allowing the measurement of the turbulent fluctuations. The diagnostic operates in the frequency range $110-130 \mathrm{GHz}$ and each channel is 100 or $200 \mathrm{MHz}$ in bandwidth $\left(B_{\mathrm{IF}}\right)$
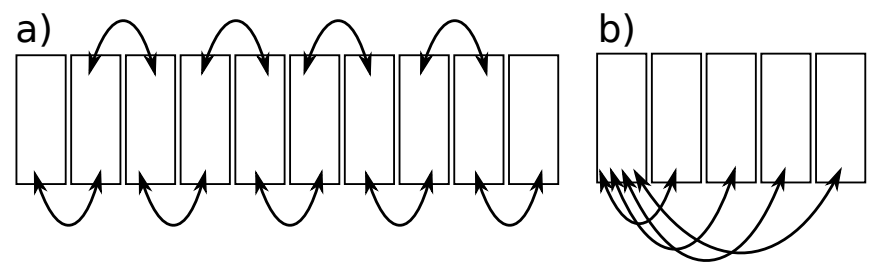

FIG. 2. Two examples of correlation patterns used to make measurements of $\delta T_{e, \perp} / T_{e}$. a) is a pattern allowing a fine resolution radial profile of $\delta T_{e \perp} / T_{e}$ and b) allows the measurement of the radial correlation length in the traditional sense.

The new CECE diagnostic on AUG allows for more detailed measurements of the ion scale electron temperature fluctuations than has been achieved before, due both to the large number of channels (28) and their arrangement in a comb pattern [24]. Rather than relying on individual pairs, as used in the previous AUG CECE diagnostic [23], where the number of radial points is $n / 2$, the comb arrangement allows $n-1$ radial points in a $\delta T_{e \perp} / T_{e}$ fluctuation profile with a radial resolution of $2-4 \mathrm{~mm}$, as shown in Figure 2 a). This arrangement also allows the possibility to measure the correlation length by correlating one channel with others at increasing separation, Figure $2 \mathrm{~b}$ ), presenting a distinct advantage over the usage of tune-able filters, in that only a single shot is required to measure both $\delta T_{e} / T$ and $L_{r}\left(T_{e}\right)$ profiles with high sensitivity.

The AUG CECE diagnostic is sensitive to low- $k$ fluctuations in the range $k_{\perp}<1.4 \mathrm{~cm}^{-1}, k_{r}<3.5 \mathrm{~cm}^{-1}$, which equates to the range $k_{\perp} \rho_{s}<0.28$ and $k_{r} \rho_{s}<0.7$, (where $\rho_{s}$ is the ion gyro-radius evaluated at the local ion sound speed and magnetic field) for a typical AUG L-mode plasma, measures in the ion-scale range. Fluctuations in this range are expected to dominate both the electron and ion heat flux $\left(Q_{e}\right.$ and $\left.Q_{i}\right)$ under most circumstances, however, it has been shown that electron scales can contribute directly to $Q_{e}$ if the Electron Temperature Gradient (ETG) mode growth rate is above $\sqrt{m_{i} / m_{e}} \simeq 60$ [17] and through the effects of cross-scale coupling [19].

Fluctuation amplitudes are calculated using Equation 1 , where $\gamma_{c}$ is the complex coherence [37] between channel pairs, $\gamma_{\mathrm{bg}}$ is the background coherence estimated from a region of frequency space separated from the fluctuations and $\Re$ denotes taking the real part. For a more in-depth discussion of the signal analysis techniques, error estimation and sensitivity limits used in this paper, see Ref [24]

$$
\left(\frac{\delta T_{e \perp}}{T_{e}}\right)^{2}=\frac{2}{B_{\mathrm{IF}}} \int_{f_{0}}^{f_{1}} \frac{\Re\left\{\gamma_{c}(f)-\gamma_{\mathrm{bg}}\right\}}{1-\Re\left\{\gamma_{c}(f)-\gamma_{\mathrm{bg}}\right\}} d f .
$$

The temperature fluctuation profile measured in the repeat discharge, together with two subsequent discharges where the channel position was scanned, are shown in Figure 3. The outer region of the plasma $\left(\rho_{\text {tor }}>0.87\right)$ has an optical depth $\tau$ below 2. Radii smaller than this are considered optically thick for our purpose, with $\tau>3$ for all further measurements presented in this paper. The sensitivity limit is $0.13 \%$ for the data averaged over a 3 second period.

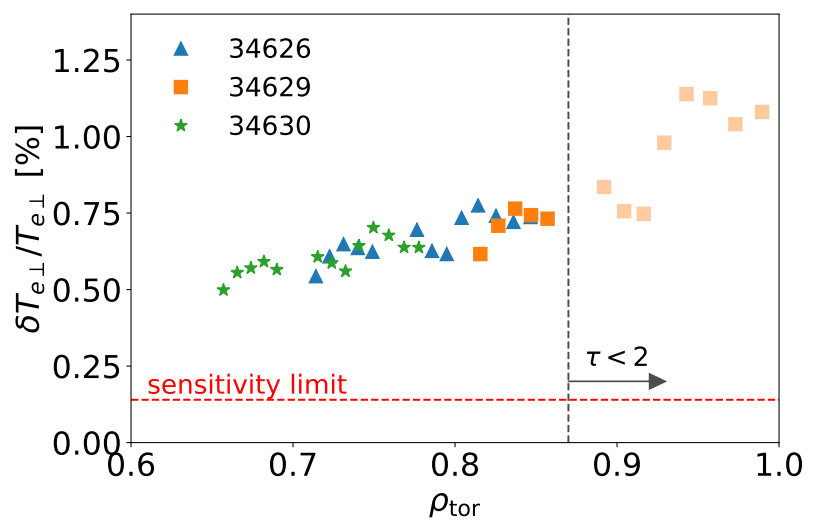

FIG. 3. $\delta T_{e \perp} / T_{e}$ profiles measured for the reference discharge 34626 and two subsequent discharges where the channel position was scanned radially inwards and outwards. The region of low optical depth $\tau$ is marked.

Figure 4 shows Equation 1 evaluated for different channel spacings, calculated from the cold resonance positions. The figure contains data from two sets of CECE channels, one with $B_{\mathrm{IF}}=100 \mathrm{MHz}$, spaced at $125 \mathrm{MHz}$ $(\sim 2 \mathrm{~mm})$ and another with $B_{\mathrm{IF}}=200 \mathrm{MHz}$, spaced at $250 \mathrm{MHz}(\sim 4 \mathrm{~mm})$. The two sets agree with each 
other very well indicating the robustness of the analysis techniques. The estimated correlation length for the $T_{\text {rad }}$ fluctuations, $L_{\text {meas }}$ is $8.4 \mathrm{~mm}\left(5.2 \rho_{s}\right)$ in this discharge, at $\rho_{\text {tor }}=0.78$. Since each ECE channel has a finite radial width $(\simeq 5 \mathrm{~mm})$, the measured correlation length $L_{r}\left(T_{e, \text { meas }}\right)$ is moderately larger than $L_{r}\left(T_{e}\right)$. The necessary modeling for the interpretation of this measurement will be discussed in section IV in the context of the comparison of radial correlation lengths to gyrokinetic simulations via a synthetic diagnostic.

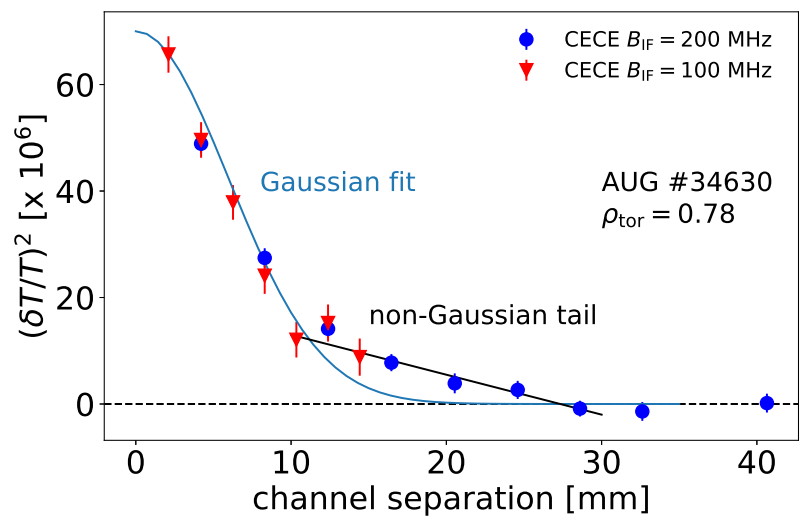

FIG. 4. The spatial correlation length function for repeat discharge $34630\left(\rho_{\text {tor }}=0.78\right)$. The correlation function has a Gaussian shape with an elongated tail. The $1 / e$ width of the Gaussian part is $8.4 \mathrm{~mm}\left(5.2 \rho_{s}\right)$

\section{B. Measurement of phase angle between $n_{e}$ and $T_{e \perp}$}

The electron heat flux arising from turbulent fluctuations depends not just on the amplitudes of $\delta T_{e}, \delta n_{e}$ and potential, $\delta \phi$, but also from the coherencies and crossphase angles between these quantities. Using a coupled ECE radiometer and reflectometer which share a line of sight to the plasma, it is possible to measure turbulent density and temeperature fluctuations at the same location [38-40]. Provided that radial alignment between the ECE radiometer channel and the reflectometer cut-off can be achieved to within a turbulent correlation length, cross-correlating the signals from the two diagnostics allows the cross-phase angle of the density and temperature fluctuations to be calculated. The reflectometer used for this study was a bi-static W band $(75-100 \mathrm{GHz}$ ) reflectometer in normal incidence, operating at fixed frequency. The reflectometer has a heterodyne receiver with in-phase (I) and quadrature (Q) detection, which are high pass filtered below $10 \mathrm{kHz}$, leaving the fluctuating I and Q components of the signal. The reflectometer signal phase is then given by $\arctan (Q / I)$ which is then unwrapped, and the signal amplitude by $\sqrt{I^{2}+Q^{2}}$.

In the following, $\alpha_{n T}$ is defined as:

$$
\alpha_{n T}=\arg \left\{S_{T_{e \perp}}^{*} S_{n_{e}}\right\}
$$
[41]:

which has a statistical uncertainty given in radians by

$$
\sigma_{\alpha_{n T}}=\sqrt{\frac{1}{2 n_{d}}}\left(\frac{1}{\left|\gamma_{c}\right|^{2}}-1\right)
$$

where $n_{d}$ is the number of independent records used to calculate $\gamma_{c}$. For the AUG CECE, the channel comb continuously covers a sufficiently large radius, that typically only one shot is required to find the peak sensitivity of the reflectometer with one of the ECE channels. The experimental strategy is to launch the reflectometer at fixed frequency (cut-off position) and correlate the reflectometer signal amplitude with all of the available ECE channels. This approach is demonstrated in Figure 5, which shows the average coherence in the range $0-100 \mathrm{kHz}$ between the reflectometer amplitude and several ECE radiometer channels (squares) taken over a period of $1 \mathrm{~s}$. The $\mathrm{x}$-axis represents the ECE channel position given in $\mathrm{mm}$ from the outermost channel $(0 \mathrm{~mm})$, increasing along the line of sight towards the plasma core. A clear maximum peak in coherence can be distinguished at $40 \mathrm{~mm}$, indicating that reflectometer 'alignment' has been found. This corresponds to $\rho_{\text {tor }}=0.744$. The triangles represent the average $\mathrm{CECE}$ coherence with reference to the ECE channel which gives the highest coherence with the reflectometer. One can see that after $44 \mathrm{~mm}$, the coherence between reflectometer and CECE falls off approximately as fast as the CECE coherence falls, whereas the coherence between reflectometer and ECE before $40 \mathrm{~mm}$ falls somewhat slower, suggesting the reflectometer cut-off has been captured. Figure 6 shows the coherence between two adjacent $\mathrm{CECE}$ channels in panel a) for two radii, which correspond to the maximal average coherence between reflectometer and CECE in two cases. Panel b) shows the coherence between reflectometer and ECE radiometer channel for these cases and panel c) shows the cross phase spectra. One can see that the phase has a relatively steady value where the coherence is high (below $50 \mathrm{kHz}$ ) and is random where the coherence is low (above $50 \mathrm{kHz}$ and below $10 \mathrm{kHz}$ ).

It has previously been shown that $\alpha_{n T}$ varies continuously with increasing/decreasing electron temperature gradient $[42,43]$. This makes $\alpha_{n T}$ a particularly interesting quantity to use in the validation of mixed turbulent mode plasmas, since it can provide a strong constraint on the mix of electron and ion modes in the saturated turbulent state, thus constraining the ratio of electron and ion mode drives as valid inputs to the simulations. 


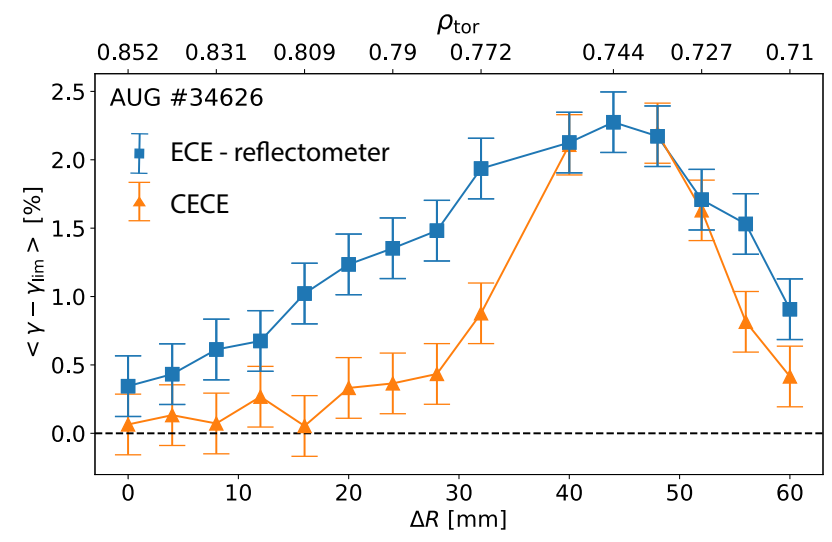

FIG. 5. Average coherence, $\left|\gamma_{c}\right|$ between reflectometer amplitude and individual CECE radiometer channels (triangles) averaged between $0-100 \mathrm{kHz}$ with noise level subtracted. This is compared to the average coherence between a fixed CECE radiometer channel at $\Delta R=44 \mathrm{~mm}$ and other CECE radiometer channels (squares), scaled by 0.45

\section{GYROKINETIC VALIDATION}

\section{A. Linear Gyrokinetic Simulations}

Linear simulations were first performed with the GENE [17] linear eigenvalue solver in order to inform the non-linear simulations. Figure 7 shows the growth rate and frequency of the two most unstable eigenmodes in simulations for $0.9,1.0$ and 1.2 times the experimentally measured ion temperature gradient scale length, $1 / L_{T_{i}, \text { nom. }}$. At the lowest value for $1 / L_{T_{i}}$, the dominant linear mode is the hybrid Trapped Electron Mode - ETG (TEM-ETG), so called in this instance, because with increasing $k$, there is no discontinuity in growth rate or frequency between ion scales and electron scales, making a definition of TEM and ETG difficult. As $1 / L_{T_{i}}$ is increased the TEM-ETG mode peak is suppressed and the Ion Temperature Gradient (ITG) mode grows, as expected, to become the fastest growing mode.

The figure shows that, within the error-bars of the experimental $1 / / L_{T_{i}}$,nom., the plasma at this radial position could either be classified as a linearly ITG or TEM. However, the non-linear mix of modes may well be different, as the ITG or TEM-ETG may be subject to different saturation mechanisms and non-linearly interact with each other.

Not shown in Figure 7, are the high $k$ values. The ratio of high and low $k$ peak growth rates $\gamma_{\text {high k }} / \gamma_{\text {low k }}$ $=35$. Previously it has been suggested that this figure of merit should be as high as $\sqrt{m_{i} / m_{e}} \simeq 60$ for the heat flux carried at the electron scales to be significant. Therefore it was decided to simulate only the ion scales in this study.
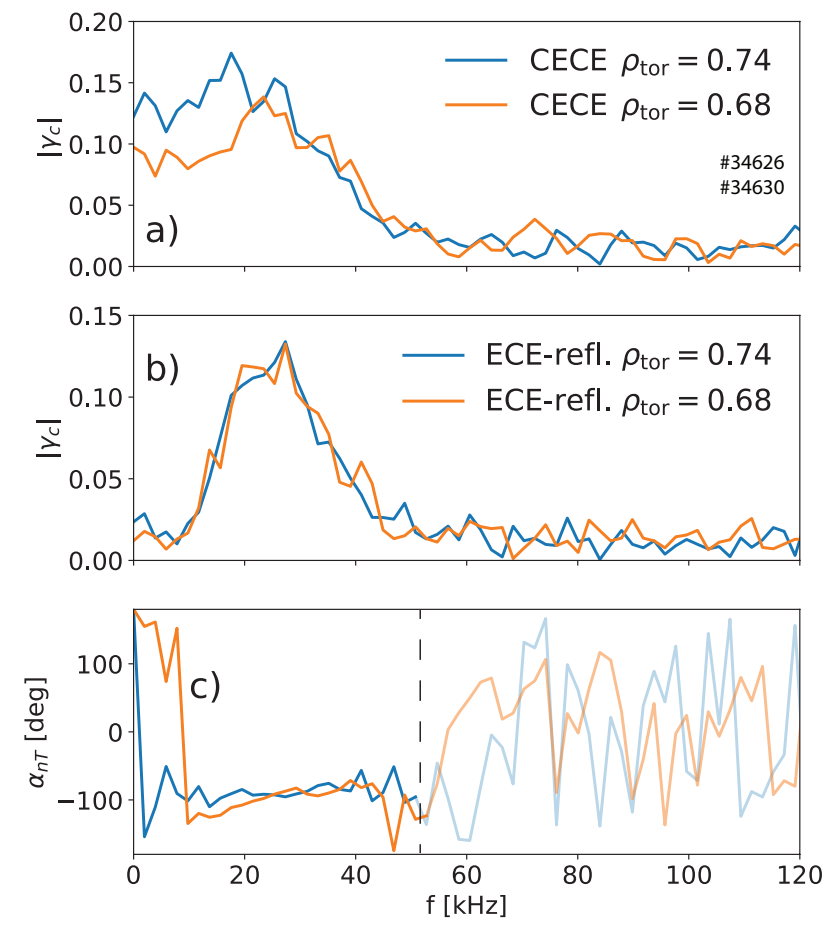

FIG. 6. a) Coherence between CECE channels at two radii corresponding to the highest coherence between CECE and reflectometer in two cases. b) Coherence between CECE and reflectometer amplitude for two different radii. c) The crossphase between reflectometer and CECE for the two radii. Values to the right of the dashed line correspond to low coherence, thus high error.

\section{B. Non-linear Gyrokinetic Simulations}

Non-linear gyrokinetic simulations were performed with GENE for the reference discharge (profiles shown in Figure 1) at $\left.\rho_{\text {tor }}=0.75\right)$. The GK simulations were ion-scale, local flux tube simulations, including electromagnetic effects, using a realistic ion/electron mass ratio and using an experimentally reconstructed magnetic geometry from the CLISTE equilibrium code. Collisions were modeled via a linearized Landau-Boltzmann operator and impurities included through a $Z_{\text {eff }}$ term in this operator. The experimental input (nominal) values are presented in Table I. Sensitivity scans are performed around these nominal values to explore the agreement / disagreement with TRANSP calculated heat fluxes. Both the ion and electron temperature gradient scale lengths are varied with the experimental error bars, in two separate sets of scans.

Figure 8 shows the GK conductive heat fluxes in the electron and ion channels compared to the conductive heat flux values inferred by the TRANSP transport analysis code (shaded regions) for the $1 / L_{T_{i}}$ scan. Both the $Q_{i}$ and $Q_{e}$ are in agreement within uncertainties with those estimated from TRANSP, for the nominal gradient 

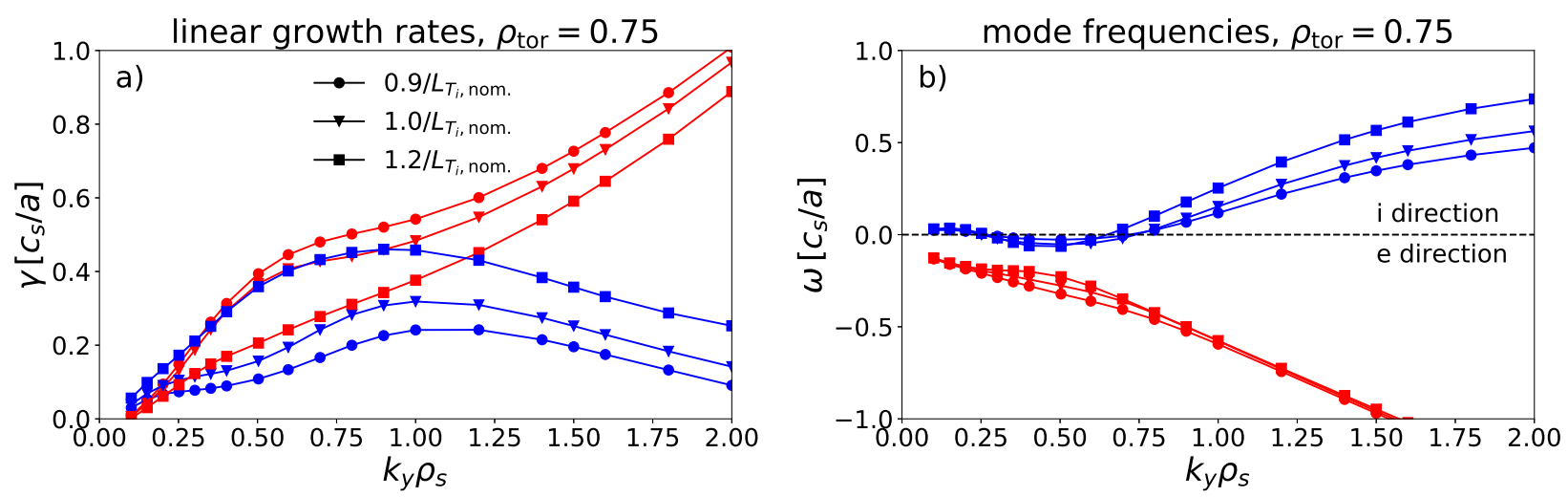

FIG. 7. The linear growth rate (a) and mode frequency (b) for the ion (blue) and electron (red) modes. The modes are shown

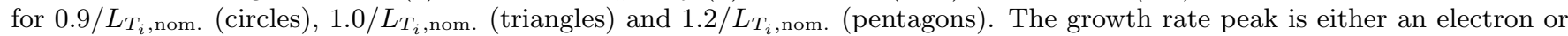
ion mode within the experimental uncertainty and is thus classed as a mixed mode plasma.

\begin{tabular}{cc}
\hline \hline Quantity & nominal value \\
\hline$I_{p}[\mathrm{MA}]$ & 1.0 \\
$\rho_{\text {tor }}$ & 0.75 \\
$q$ & 2.28 \\
$\hat{s}$ & 1.923 \\
$\hat{s}_{E \times B} L_{\text {ref }} / c_{s}$ & 0.0168 \\
$1 / L_{T_{i}}=-d / d \rho_{\text {tor }}\left(\ln T_{e}\right)$ & 5.11 \\
$1 / L_{T_{e}}=-d / d \rho_{\text {tor }}\left(\ln T_{i}\right)$ & 2.65 \\
$1 / L_{n_{e}}=-d / d \rho_{\text {tor }}\left(\ln n_{e}\right)$ & 1.46 \\
$T_{e}[\mathrm{keV}]$ & 0.604 \\
$n_{e}\left[10^{19} m^{-3}\right]$ & 1.53 \\
$T_{i} / T_{e}$ & 0.647 \\
$Z_{\text {eff }}$ & 1.6 \\
$\beta_{e}[\%]$ & 0.0539 \\
$\nu_{e i} /\left(L_{\text {ref }} / c_{s}\right)$ & 0.370 \\
$R_{\text {axis }}[\mathrm{m}]$ & 1.65 \\
$a[\mathrm{~m}]$ & 0.47 \\
$B_{\text {ref }}[\mathrm{T}]$ & 2.626 \\
$L_{\text {ref }}[\mathrm{m}]$ & 0.6523 \\
\hline \hline
\end{tabular}

TABLE I. The input values for the nominal case for both the linear and non-linear simulations. $\hat{s}$ is the magnetic shear and $\hat{s}_{E \times B}$ is the $E \times x B$ shearing rate

inputs. A reduction of $1 / L_{T_{i}}$ of $5 \%$ brings the simulations within $5 \%$ of the TRANSP values for $Q_{i}$ and $Q_{e}$. Scans of $1 / L_{T_{e}}$ were also performed at fixed $1 / L_{T_{i}}$ and one of those points $\left(0.87 / L_{T_{e}, \text { nom. }}\right)$ is shown in Figure 8 (squares). This will be of particular interest later as it provides the best overall match to the measured turbulent quantities.

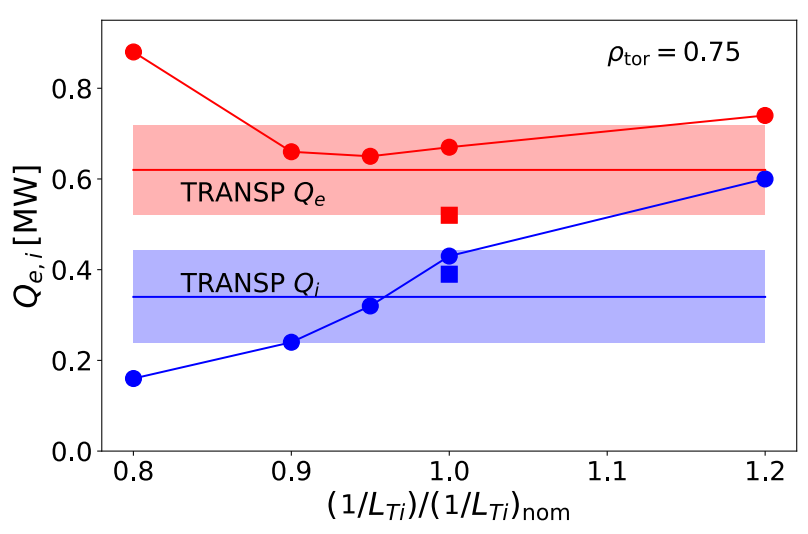

FIG. 8. Inferred experimental heat fluxes from TRANSP at $\rho_{\text {tor }}=0.75$. Ions and electrons are represented by horizontal lines and the uncertainty by the shaded regions. GK conductive heat fluxes are over-plotted for both species as a function of the change in $1 / L_{T_{i}}$. Additional point (squares) show $Q_{i}$ and $Q_{e}$ for $1.00 / L_{T_{i}, \text { nom. }}, 0.87 / L_{T_{e}, \text { nom. }}$

\section{CECE synthetic diagnostic}

Critical to any direct quantitative comparison of the $T_{e}$ fluctuation measurements to GK simulations requires the use of a sufficiently accurate synthetic diagnostic to apply to the simulation output data [10]. This synthetic diagnostic ideally should not be a source of error for the quantitative comparison. In our case, GK fluctuation data has the toroidal velocity as measured from CXRS imposed on it and is then mapped to $\mathrm{R}, \mathrm{Z}$ coordinates. The perpendicular velocity of the structures is then found to agree well with that measured by the poloidal correlation reflectometer [44] at this radius. There are several effects that are significant for the fluctuation amplitude calculated by the synthetic diagnostic. 
Of prime importance is the accurate modeling of the spatial region to which the CECE is sensitive. The finite volume from which the measurements are made results in a spatial smoothing, which dictates the $k$ range to which the diagnostic is sensitive [45]. Since, in the simulations, there is significant fluctuation power above the measured $k$ range, this can have a significant impact on the predicted fluctuation amplitude. A 2D Gaussian function in the $R, z$ plane is used to model the finite volume of the real measurements. The radial size of the Gaussian function is taken from a fit to the emissivity function [46], determined using a standard ECE radiation transport model (ECFM) [47]. This model takes into account the finite bandwidth of the IF filters $\left(B_{\mathrm{IF}}\right)$, the relativistic broadening and ECE frequency downshift, the Doppler broadening at finite toroidal angle and beam refraction in the plasma. This synthetic diagnostic currently uses a fitted Gaussian with 1/e radial width of $4.7 \mathrm{~mm}$.

The $1 / e$ width of the Gaussians in the $z$ direction, $w_{z}$, are set by the quasi-optical beam width and are critical parameters for the synthetic diagnostic. The beam width was measured using a near-field measuring technique ex-situ. This technique measures the complex field in two polarizations in the near field of the diagnostic antenna and then extrapolates the field in space using a Fast Irregular Antenna Field Transformation Algorithm [48]. The advantage of this measurement technique is that the beam waist may be extrapolated reliably to arbitrary distances from the CECE optics. Two sets of measurements were made over the range $75-105 \mathrm{GHz}$. Firstly, the antennas were measured alone and a Gaussian beam was fit to the results. This was then used as input to a Gaussian Optics (GO) formalism [49] to propagate the beam through the focussing elements. Secondly, the beam from the complete CECE optics was measured and compared directly to the GO predictions. Figure 9 shows the results of this comparison (blue line - GO, green stars - measurement). The trends in the antenna waist and position vs. frequency were then extrapolated to the measurement frequency of $117.5 \mathrm{GHz}$ and a new beam was calculated (solid red line in Figure 9 ). Since this extrapolation comes with some uncertainty, the results for $\pm 20 \%$ of the extrapolated antenna waist size are also shown (red dashed and dotted respectively). The CECE measurements compared to GK simulations in this paper were made in the AUG plasma at a position $515 \mathrm{~mm}$ from the mirror.

The plasma considered in this study is sufficiently optically thick $(\tau>3)$ that contributions from density fluctuations are negligible. Further, since the CECE is only sensitive to the perpendicular temperature fluctuations [36], the synthetic diagnostic only acts on those. In the non-linear simulations performed in the study presented here, the $\delta T_{e \perp}$ is found to be a factor of 3.8 higher than $\delta T_{e \|}$. Since $\delta T_{\text {tot }}=2 / 3 \delta T_{\perp}+1 / 3 \delta T_{\|}, \delta T_{e \perp}$ is thus $33 \%$ higher than $\delta T_{e, \text { tot }}$. There has only been one other gyrokinetic validation study thus far where this effect has been taken into account, significantly improving agree-

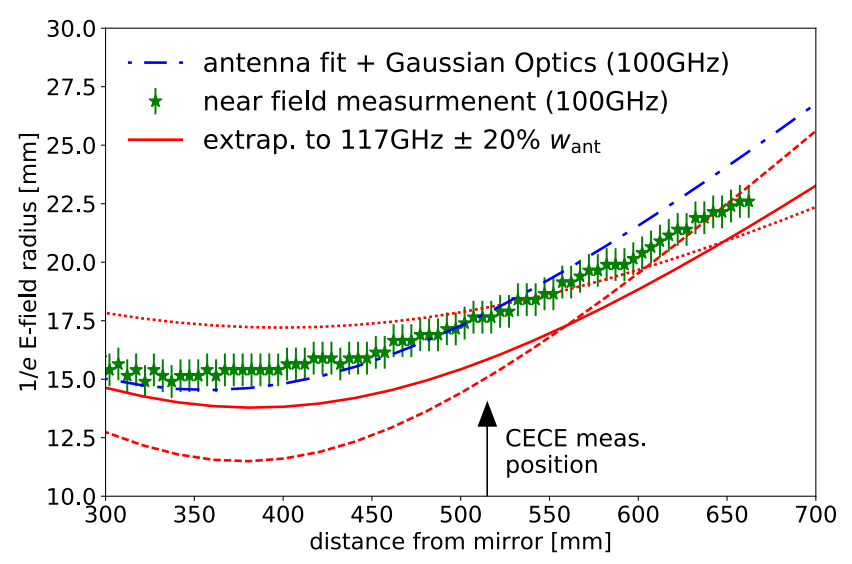

FIG. 9. Measurements and Gaussian Optics (GO) model comparisons for the beam waist size after the focussing mirror. Stars represent measurements at $100 \mathrm{GHz}$, the dash-dot line is the GO result at $100 \mathrm{GHz}$. The GO results at $117.5 \mathrm{GHz}$ for extrapolated antenna parameters are shown (solid line) and $\pm 20 \%$ antenna beam waist size (dashed, dotted respectively). The CECE measurements compared to GK simulations were performed at $515 \mathrm{~mm}$ from the antenna.

ment between the code and the experimental measurements in that case[6].

For the simulation of the measurement of $\alpha_{n T}$, the CECE synthetic diagnostic Gaussian filters are applied simultaneously to the $\delta T_{e \perp}$ and $\delta n_{e}$ fields and crosscorrelations are performed on the time series resulting from co-located Gaussian filters in a similar manner to the synthetic diansotic used previously on GYRO simulations [39]. The justification is that the CECE limits the k-range over which the measurements are made more than the reflectometer in the poloidal direction. Reflectometer physics is not explicitly included and the Born approximation is assumed to apply to the fluctuation amplitude. There is experimental evidence for this assumption, both from our measurements and in previous experiments at DIII-D [40].

\section{Comparison of simulations to measured fluctuations}

Comparing $\delta T_{e \perp} / T_{e}$ from GK simulations of the reference discharge to the measurements shows that the temperature fluctuations are systematically over-estimated, even when the ion and electron heat fluxes are matched within error bars. Two example spectra are shown in Figure 10, with the experimental measurements in black and the synthetic diagnostic result in orange. The GK spectrum is both higher in amplitude and broader in frequency than in the experiment with $\left(\delta T_{e \perp} / T_{e}\right)_{\text {expt. }}=$ $0.70 \%$ and $\left(\delta T_{e \perp} / T_{e}\right)_{\mathrm{GK}}=1.14 \%$. Increasing the CECE beam width by $30 \%$, which is greater than the experimentally measured uncertainty, cannot account for the discrepancy, in fact a factor of 2 is required to find agree- 
ment between experiment and simulation.

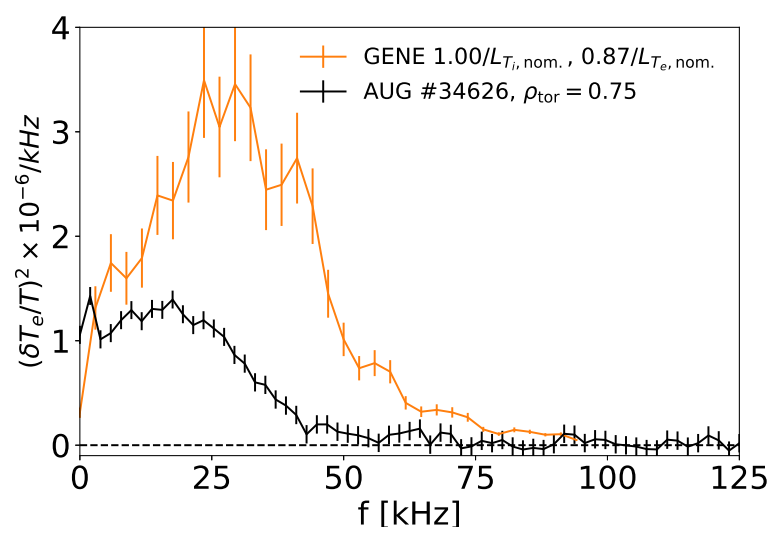

FIG. 10. A comparison of the experimentally measured $(\delta T / T)^{2}$ spectrum and that predicted by the GK simulations. The GK simulations significantly overestimates the fluctuation amplitude.

In contrast to this, the correlation function is found to agree well in shape. Correlation lengths are calculated by fitting Gaussian functions to both the experimental data and GK data. In this case $L_{r}\left(T_{e \perp}\right)_{\mathrm{GK}}=10.5 \mathrm{~mm}$ and $L_{r}\left(T_{e \perp}\right)_{\text {expt. }}=9.8 \mathrm{~mm}$. There is of course some ambiguity as neither the GK correlation function or the experimentally measure one are strictly Gaussian, both possessing elevated tails. The data in Figure 4, which is made at higher resolution, slightly further out of an identical plasma shows this more clearly for the case of the experiment. It is these fitted values which are used in the validation metric in Section IVE, with a representative error of $1.5 \mathrm{~mm}$.

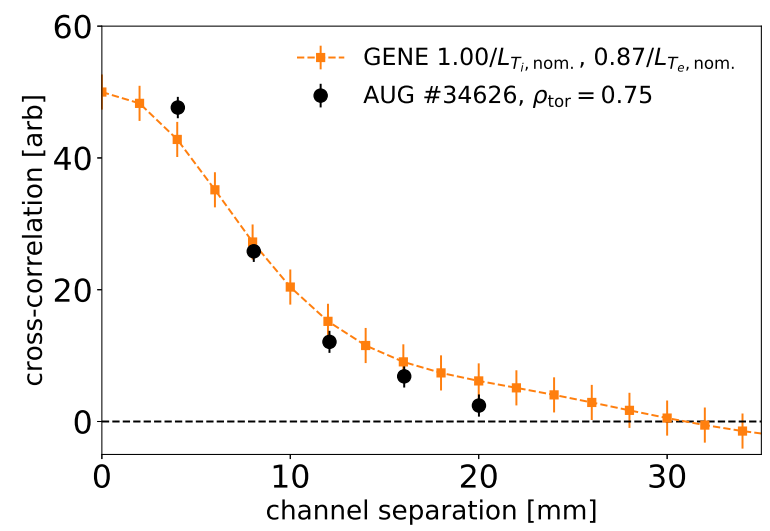

FIG. 11. Measured spatial correlation function with reference to a CECE channel at $\rho_{\text {tor }}=0.75$ (triangles), and the synthetic diagnostic result for reduced $1 / L_{T_{e}}$ (orange dashed line). Again remarkable agreement can be found between the synthetic diagnostic results and the experiment.

For the same simulation there is also quantitative agreement between measured and simulated $\alpha_{n T}$. Figure 12 shows the cross-phase frequency spectra measured in the repeat discharge and the GK simulated result. In the region where there is high coherence between reflectometer and CECE $(10-50 \mathrm{kHz})$, there is quantitative agreement in the cross-phase.

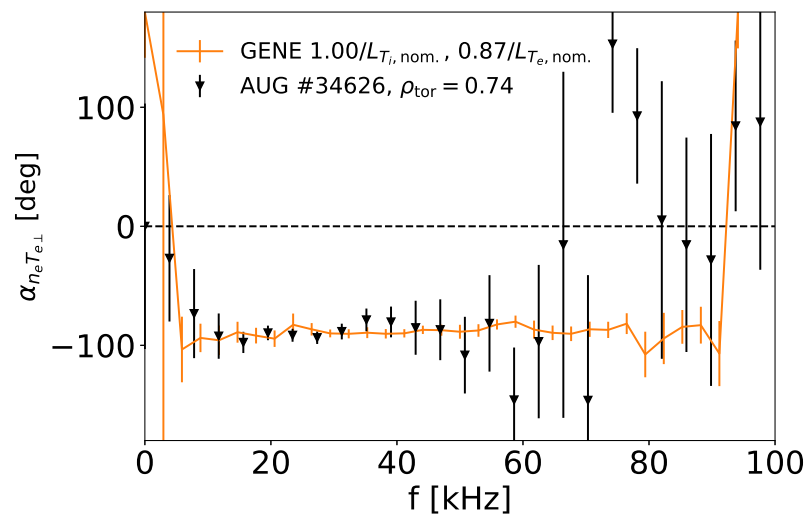

FIG. 12. Experimentally measured $\alpha_{n T}$ spectrum (triangles) at maximum coherence $\left(\rho_{\text {tor }}=0.74\right)$ compared to that from the synthetic diagnostic for reduced $1 / L_{T_{e}}$ (solid line). Remarkable quantitative agreement is found in this case.

The $\alpha_{n T}$ cross-phase angle can be a very useful discriminator between simulations which differ in the ion and electron mode content. This can be seen in Figure 13, which shows the synthetic diagnostic results for selected points in the sensitivity scans performed. The squares show the result of an $1 / L_{T_{i}}$ scan with $1 / L_{T_{e}}$ fixed at the nominal value. The triangles are two points of an $1 / L_{T_{e}}$ scan with $1 / L_{T_{i}}$ fixed at the nominal value. The black point is the experimentally measured $\alpha_{n T}$. For reference in this figure, artificially setting $1 / L_{T_{e}}=0$ in the simulations (ITG like) gives $\alpha_{n T}=-135^{\circ}$ and setting $1 / L_{T_{i}}=0$ (TEM like) gives $\alpha_{n T}=-15^{\circ}$. It can be seen that increasing $1 / L_{T_{i}}$ smoothly increases the magnitude of the phase angle towards the ITG like value as the turbulence becomes more ITG like in nature. In contrast, increasing $1 / L_{T_{e}}$, decreases the magnitude of $\alpha_{n T}$, becoming more TEM like. The experimental point is in quantitative agreement for the simulations with a stronger ITG drive compared to TEM drive.

The direct comparisons of fluctuating quantities presented in this section in Figures 10, 11 and 12 come exclusively from a single GK simulation using $1.0 / L_{T_{i}, \text { nom. }}, 0.87 / L_{T_{e}, \text { nom. }}$. This simulation gives the closest agreement for each of the turbulent quantities and is within experimental uncertainties for $Q_{i}$ and $Q_{e}$. The metrics which justify this simulation choice for the comparisons are discussed in section IVE. 


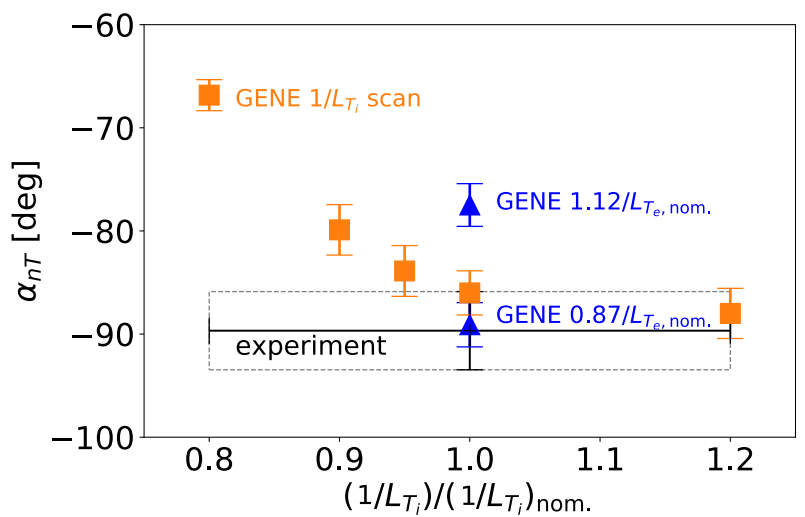

FIG. 13. The experimentally measured $\alpha_{n T}$ agrees quantitatively for those simulations which have either less electron mode drive or more ion mode drive than nominal.

\section{E. Validation metric}

It is useful to adopt a metric that allows an asessment of the overall quality of the simulations when all the experimental comparisons are considered. The parmeters constraining the simulations in this study are: $Q_{i}, Q_{e}$, $\delta T_{e \perp} / T_{e}, L_{r}\left(T_{e \perp}\right)$ and $\alpha_{n T}$. The choice of validation metric is an ongoing discussion in the field of validation with various mathematical formulations proposed $[21,50,51]$ and a recent review article [52] on the subject. In this study, the formulation laid out by Ricci et al [51] has been employed. First an "uncertainty-normalized distance", $d_{i}$ is calculated for each observable. This quantifies the distance between the simulated quantity, $s_{i}$ and the experimental quantity, $e_{i}$ in units of the uncertainties $\Delta s_{i}$ and $\Delta e_{i}$ :

$$
d_{i}=\sqrt{\frac{\left(s_{i}-e_{i}\right)^{2}}{\Delta s_{i}^{2}+\Delta e_{i}^{2}}}
$$

where the sum over instances has been dropped, since in this case we want to calculate values for each individual simulation and there is only one measurement of each experimental quantity. The values of $d_{i}$ for each of the observable for each of the simulations performed can be found in Table II. and bar charts of $d_{i}$ can be seen in Figure 14 for the 4 flux matching simulations.

A bounded error metric $R$ can then be defined

$$
R_{i}=\frac{1+\tanh \left[\left(d_{i}-d_{0}\right) / \lambda\right]}{2},
$$

where $R_{i}=0$ indicates perfect agreement and $R_{i}=1$ indicates complete disagreement. The free parameter $d_{0}$ defines the level at which transition from agreement to disagreement and is set to 1.5 , meaning that a $d_{i}$ of 1.5 maps to an $R_{i}$ of 0.5 . The parameter $\lambda$ defines the sharpness of the transition from agreement to disagreement
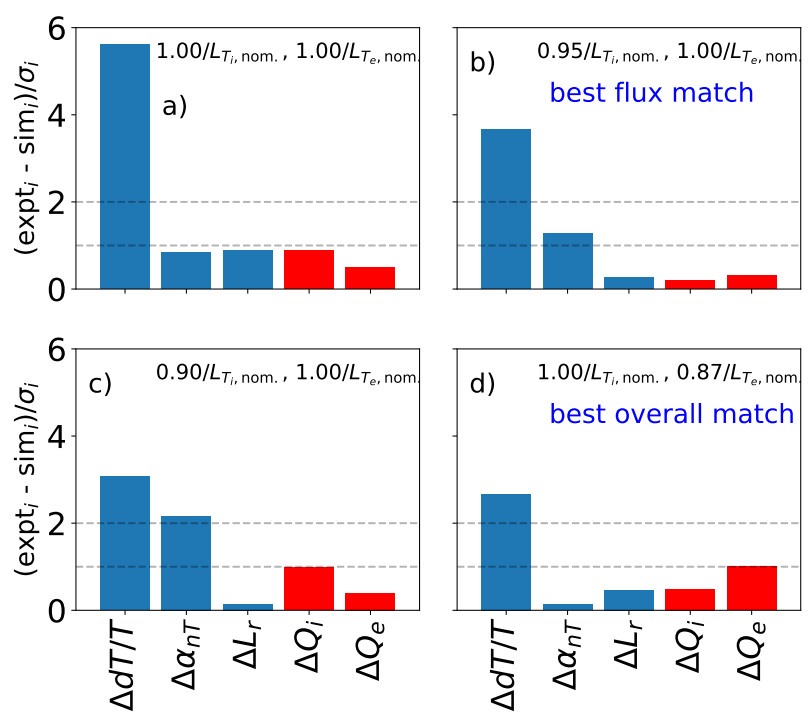

FIG. 14. Bar charts showing the uncertainty distance $d_{i}$ for all compared observables for four selected cases. Each case agrees with both $Q_{i}$ and $Q_{e}$ within uncertainties and the best match to the turbulence properties is for (1.0/ $\left./ L_{T_{i}, \text { nom. }}, 0.87 / L_{T_{e}, \text { nom. }}\right)$

and is taken to be 0.5 in the below analysis. These values were suggested in [51] as reasonable choices and it was shown that the conclusions drawn for this metric are not significantly affected by the precise values of $d_{0}$ and $\lambda$, provided $1 \leq d_{0} \leq 2$ and $0.1 \leq \lambda \leq 1$. A combined metric can then be defined which gives a single value bounded between 0 and 1 for the simulation as a whole. The combined metric is given as

$$
\chi=\frac{\sum_{i=1}^{N} R_{i} H_{i} S_{i}}{\sum_{i=1}^{N} H_{i} S_{i}},
$$

where $H_{i}=1 / h_{i}$ quantifies the level of the measurement in the primacy hierarchy [50]. In this study $h_{i}=1$ for a measured fluctuation quantity and $h_{i}=2$ for a heat flux. $S_{i}$ is the sensitivity function and is a decreasing function of the experimental and simulation uncertainties, defined as

$$
S_{i}=\exp \left[-\frac{\left(\Delta s_{i}+\Delta e_{i}\right)}{\left(\left|s_{i}\right|+\left|e_{i}\right|\right)}\right] .
$$

The validation metric allows one to compare the simulations in a defined and quantitative manner. Simulations with $\chi<0.5$ are defined to be in overall agreement with the experiment and with $\chi>0.5$ in disagreement [51]. Figure 15 shows $\chi$ for the simulation set presented in this paper. A minimum $\chi$ (best agreement) is found for the simulation performed at $1.0 / L_{T_{i}, \text { nom. }}, 0.87 / L_{T_{e}, \text { nom. }}$, which is also the best overall match to the turbulence measurements and this is 


\begin{tabular}{cccccccc}
\hline \hline$L_{T_{i}, \text { nom. }} / L_{T_{i}}$ & $L_{T_{e}, \text { nom. }} / L_{T_{e}}$ & $d_{Q i}$ & $d_{Q e}$ & $d_{\delta T / T}$ & $d_{L r}$ & $d_{\alpha}$ & $\chi$ \\
\hline 0.80 & 1.00 & 1.76 & 2.62 & 2.66 & 0.02 & 5.60 & 0.71 \\
0.90 & 1.00 & 0.98 & 0.40 & 3.07 & 0.15 & 2.17 & 0.51 \\
0.95 & 1.00 & 0.20 & 0.30 & 3.66 & 0.27 & 1.28 & 0.33 \\
1.00 & 1.00 & 0.88 & 0.50 & 5.61 & 0.89 & 0.84 & 0.30 \\
1.00 & 1.12 & 0.78 & 2.52 & 3.94 & 0.90 & 2.82 & 0.65 \\
1.20 & 1.00 & 2.55 & 1.21 & 5.21 & 0.05 & 0.37 & 0.40 \\
1.00 & 0.87 & 0.49 & 1.01 & 2.67 & 0.46 & 0.13 & 0.27 \\
\hline \hline
\end{tabular}

TABLE II. Uncertainty normalised distance, $d$, and validation metric, $\chi$, for all simulations in the $1 / L_{T_{i}}$ and $1 / L_{T_{e}}$ scans considered in this study.

a different simulation to that which best matches the heat fluxes $\left(0.95 / L_{T_{i}, \text { nom. }}, 1.0 / L_{T_{e}, \text { nom. }}\right)$. Although both simulations are in agreement with the heat fluxes, caution should be exercised in concluding that the best heat flux match is the most physically realistic simulation and highlights the importance of having as many measurements as possible to constrain the simulations. There is a clearly defined minimum in $\chi$ as a function of $1 / L_{T_{i}}$, with only a small improvement when reducing $1 / L_{T_{e}}$ by $12 \%$. In contrast, increasing $1 / L_{T_{e}}$ by $12 \%$ brings a significant penalty to the overall agreement. This is brought about by a large discrepancy arising in all three of $Q_{e}$, $\alpha_{n T}$ and $L_{r}\left(T_{e}\right)$. Similar to Ricci, we also find that conclusions based primarily on the relative ordering of the $\chi \mathrm{s}$ of the simulations, i.e. which simulation proved a closer match to experiment is not affected by the exact choice of $d_{0}$ and $\lambda$ within the stated ranges.

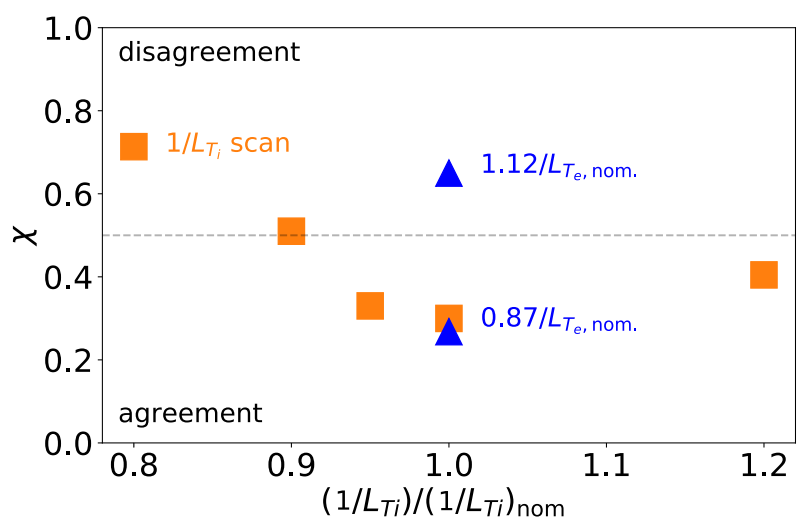

FIG. 15. A validation metric, $\chi$ for assessing the quality of the simulations. The dashed line at $\chi=0.5$ marks the boundary between overall agreement and disagreement.

It is of note that the simulations performed at $1.0 / L_{T_{i}, \text { nom. }}, 0.87 / L_{T_{e}, \text { nom. }}$ and $1.0 / L_{T_{i}, \text { nom. }}, 1.0 / L_{T_{e}, \text { nom. only differ a little in their }}$ respective $\chi_{\mathrm{s}}$, even though one can see in figure 14 that the disagreement in $\delta T / T$ at the nominal parameters is significantly larger than at $1.0 / L_{T_{i}, \text { nom. }}, 0.87 / L_{T_{e}, \text { nom. }}$. This is because $0.98>R_{i}>1$ for $d_{i}>2.5$ so large disagreements are not sufficiently distinguished. This means that the $\chi$ metric also does not distinguish very well between simulations with parameters which all disagree strongly with experiment. However, this may be seen as an advantage as this behaviour also prevents one strongly disagreeing quantity from dominating agreement in the other quantities.

\section{SUMMARY AND CONCLUSIONS}

New measurements of turbulent electron temperature fluctuation amplitude $\delta T_{e \perp} / T_{e}$, radial correlation length $L_{r}\left(T_{e \perp}\right)$ and density-temperature phase angle $\alpha_{n T}$ have been made with an upgraded Correlation ECE diagnostic [24] and nT-phase diagnostic on ASDEX Upgrade. The new diagnostic uses a closely spaced channel comb to allow measurement of high radial resolution $\delta T_{e \perp} / T_{e}$ and $L_{r}\left(T_{e \perp}\right)$ profiles. The coupling of a reflectometer with the ECE radiometer allows the simultaneous measurement of density and temperature fluctuation fields and thus the measurement of $\alpha_{n T}$. The CECE channel comb gives the advantage that one can reliably capture the maximal coherence between ECE and reflectometer, making the interpretation of the measurements easier.

These measurements have been used to simultaneously constrain a set of non-linear, ion-scale gyrokinetic simulations, modeling a ECRH heated L-mode plasma $(\# 33585)$ at $\rho_{\text {tor }}=0.75$. The simulations are based on a reference discharge for which $\delta T_{e \perp} / T_{e}$ measurements are available, and a repeat discharge was performed (\#34626) to obtain measurements of $L_{r}\left(T_{e \perp}\right)$ and $\alpha_{n T}$. These values were predicted ahead of the experiment, applying the synthetic diagnostic to the reference simulation set. Although $\delta T_{e \perp} / T_{e}$ is found to be consistently over-predicted, simultaneous agreement is found for $Q_{i}, Q_{e}, L_{r}\left(T_{e \perp}\right)$ and $\alpha_{n T}$ in two of the simulations performed, and the disagreement in $\delta T_{e \perp} / T_{e}$ is minimised for the $1.0 / L_{T_{i}, \text { nom. }}, 0.87 / L_{T_{e}, \text { nom. }}$ case.

The consistent disagreement between the measured and simulated $\delta T_{e \perp} / T_{e}$ remains an outstanding issue. Initially it would seem that the simplest solution to the discrepancy is that the beam size $w_{z}$ is incorrect with respect to the experiment. It can be shown using the syn- 
thetic diagnostic that no significant effect of $w_{z}$ is found on the measurement of $\alpha_{n T}$ or $L_{r}\left(T_{e}\right)$. However, careful measurements of the CECE beam pattern were performed, and sensitivity scans were made using the CECE synthetic diagnostic, varying $w_{z}$ within the uncertainties of the measurements (20\%). It was found that this alone cannot explain the discrepancy. In order to achieve quantitative agreement in $\mathrm{n} \delta T_{e \perp} / T_{e}$ between experiment and GK, a factor of 2.2 increase in $w_{z}$ is required, 6 times greater than the measured uncertainty in $w_{z}$.

The simulations display a high degree of realism, matching conductive heat fluxes to the experiment and demonstrating low $(10-30 \mathrm{~kW})$ convective heat fluxes consistent with the negligible convective heat fluxes in the experiment. However, although parameter scans have been performed, an exhaustive scan of all the inputs to the GK code within experimental uncertainties is not practically achievable. We cannot explicitly rule out a combination of inputs within uncertainties which may satisfy all the experimental constraints. This search could be guided in the future by reduced models.

We may also consider what additional experimental constraints would give insight into this discrepancy. For the simulations to match $Q_{e}$ and be low on $\delta T_{e} / T_{e}$ means that there must be other unmeasured quantities which are also not matched. For example, measurements of the $\operatorname{deltaT}_{e}$ and $\delta n_{e}$ fluctuation $k$-spectrum and high- $k$ fluctuation amplitudes would experimentally constrain the importance of smaller scales. Measurements of $\delta n_{e} / n_{e}$, which were not available for these discharges, would also provide a strong constraint in combination with $\delta T_{e} / T_{e}$ as they are more closely related to the unmeasured potential fluctuations.

An uncertainty metric, as defined in [51] has been used to attempt to quantify the agreement of individual simulations in a sensitivity scan to the experimental constraints and it is found that a clear minimum exists at $1.0 / L_{T_{i}, \text { nom. }}, 0.87 / L_{T_{e}, \text { nom. }}$, with the simulations becoming increasingly unrealistic away from this point. For the best matched simulations, agreement is found with four of the five experimental constraints within the uncertainties.

Overall the Ricci parameter $\chi$ provides a useful framework for assessing the relative agreement between these simulations. In particular, it clearly reveals that the heat fluxes, although important, may not be the best parameters with which to validate a gyrokinetic model. As an example, the simulation performed at $0.95 / L_{T_{i}, \text { nom. }}, 1.0 / L_{T_{e}, \text { nom. provides the best match for }}$ the experimental heat fluxes, and it thus tempting to say that it is the most physically realistic. However, it is not such a good match to the measured turbulence quantities as the simulation at $1.0 / L_{T_{i}, \text { nom. }}, 0.87 / L_{T_{e}, \text { nom. }}$. When we codify the relative importance of both the position in the hierarchy of the measured constraints and how uncertain the relative measurements are (the heat fluxes are both derived and relatively uncertain) we see that it is in fact not the best representation in this framework. The GK data presented in this paper all come from the simulation with the lowest Ricci parameter and are not hand-picked for their agreement to any single quantity, demonstrating a rigorous and fair method for presenting the data.

\section{ACKNOWLEDGMENTS}

This work is supported by the US DOE under grants DE-SC0006419 and DE-SC0017381, and was performed in the framework of the Helmholtz Virtual Institute on Plasma Dynamical Processes and Turbulence Studies using Advanced Microwave Diagnostics. It has also been carried out within the framework of the EUROfusion Consortium and has received funding from the Euratom research and training programme 2014-2018 under grant agreement No 633053. The views and opinions expressed herein do not necessarily reflect those of the European Commission.
[1] J. Connor and H. Wilson, Plasma Physics and Controlled Fusion 36, 719 (1994).

[2] W. Horton, Reviews of Modern Physics 71, 735 (1999).

[3] T. Hahm, Physics of Fluids 31, 2670 (1988).

[4] A. E. White, L. Schmitz, G. R. McKee, C. Holland, W. A. Peebles, T. A. Carter, M. W. Shafer, M. E. Austin, K. H. Burrell, J. Candy, J. C. DeBoo, E. J. Doyle, M. A. Makowski, R. Prater, T. L. Rhodes, G. M. Staebler, G. R. Tynan, R. Waltz, and G. Wang, Physics of Plasmas 15, 056116 (2008).

[5] A. Casati, T. Gerbaud, P. Hennequin, C. Bourdelle, J. Candy, F. Clairet, X. Garbet, V. Grandgirard, Ö. D. Gürcan, S. Heuraux, G. T. Hoang, C. Honoré, F. Imbeaux, R. Sabot, Y. Sarazin, L. Vermare, and R. Waltz, Physical Review Letters 102, 165005 (2009).

[6] T. Görler, A. E. White, D. Told, F. Jenko, C. Holland, and T. L. Rhodes, Physics of Plasmas 21, 122307 (2014).

[7] T. Happel, A. B. Navarro, G. Conway, C. Angioni, M. Bernert, M. Dunne, E. Fable, B. Geiger, T. Görler, F. Jenko, R. McDermott, F. Ryter, and U. Stroth, Physics of Plasmas 22, 032503 (2015).

[8] M. Nakata, M. Honda, M. Yoshida, H. Urano, M. Nunami, S. Maeyama, T.-H. Watanabe, and H. Sugama, Nuclear Fusion 56, 086010 (2016).

[9] A. B. Navarro, T. Happel, T. Görler, F. Jenko, J. Abiteboul, A. Bustos, H. Doerk, and D. Told, Physics of Plasmas 22, 042513 (2015), arXiv:arXiv:1501.05163v1.

[10] C. Holland, A. White, G. McKee, M. Shafer, J. Candy, R. Waltz, L. Schmitz, and G. Tynan, Physics of Plasmas 16, 052301 (2009).

[11] A. White, N. Howard, M. Greenwald, M. Reinke, C. Sung, S. G. Baek, M. Barnes, J. Candy, A. Dominguez, 
D. Ernst, C. Gao, A. Hubbard, J. Hughes, Y. Lin, D. R. Mikkelsen, F. I. Parra, M. Porkolab, J. Rice, J. Walk, S. J. Wukitch, and A. C. Team, phys. plasmas 20 (2013), 10.1063/1.4803089.

[12] L. Lin, M. Porkolab, E. Edlund, J. Rost, M. Greenwald, N. Tsujii, J. Candy, R. Waltz, and D. Mikkelsen, Plasma Physics and Controlled Fusion 51, 065006 (2009).

[13] N. Howard, A. White, M. Reinke, M. Greenwald, C. Holland, J. Candy, and J. Walk, Nuclear Fusion 53, 123011 (2013).

[14] C. Sung, A. White, D. Mikkelsen, M. Greenwald, C. Holland, N. Howard, R. Churchill, and C. Theiler, Physics of Plasmas 23, 042303 (2016).

[15] A. Creely, N. Howard, P. Rodriguez-Fernandez, N. Cao, A. Hubbard, J. Hughes, J. Rice, A. White, J. Candy, G. Staebler, G. Conway, S. Freethy, and C. Sung, Physics of Plasmas 24, 056104 (2017).

[16] W. Dorland, F. Jenko, M. Kotschenreuther, and B. Rogers, Physical Review Letters 85, 5579 (2000).

[17] F. Jenko and W. Dorland, Plasma Physics and Controlled Fusion 43, A141 (2001).

[18] T. Görler and F. Jenko, Physics of Plasmas 15, 102508 (2008).

[19] N. Howard, C. Holland, A. White, M. Greenwald, J. Candy, and A. Creely, Physics of Plasmas 23, 056109 (2016).

[20] S. Maeyama, T.-H. Watanabe, Y. Idomura, M. Nakata, A. Ishizawa, and M. Nunami, Nuclear Fusion 57, 066036 (2017).

[21] M. Greenwald, Physics of Plasmas 17, 058101 (2010).

[22] U. Stroth, A. Bañón Navarro, G. Conway, T. Görler, T. Happel, P. Hennequin, C. Lechte, P. Manz, P. Simon, A. Biancalani, E. Blanco, C. Bottereau, F. Clairet, S. Coda, T. Eibert, T. Estrada, A. Fasoli, L. Guimarais, Ö. Gürcan, Z. Huang, F. Jenko, W. Kasparek, C. Koenen, A. Krämer-Flecken, M. Manso, A. Medvedeva, D. Molina, V. Nikolaeva, B. Plaum, L. Porte, D. Prisiazhniuk, T. Ribeiro, B. Scott, U. Siart, A. Storelli, L. Vermare, and S. Wolf, Nuclear Fusion 55, 083027 (2015).

[23] S. J. Freethy, G. D. Conway, I. Classen, A. J. Creely, T. Happel, A. Köhn, B. Vanovac, and A. E. White, Review of Scientific Instruments 87, 11E102 (2016).

[24] A. J. Creely, W. Burke, R. Leccacorvi, W. Parkin, D. Terry, A. White, G. Conway, S. Freethy, and the ASDEX Upgrade Team, Rev. Sci. Instrum. submitted.

[25] T. Happel, G. Conway, W. Kasparek, B. Plaum, C. Lechte, D. Wagner, U. Stroth, and t. A. U. Team, Proc. 10th International Reflectometry Workshop (2011).

[26] P. Hennequin, C. Honoré, A. Truc, A. Quéméneur, C. Fenzi-Bonizec, C. Bourdelle, X. Garbet, G. Hoang, and t. T. S. Team, Nuclear Fusion 46, S771 (2006).

[27] T. Happel, T. Görler, P. Hennequin, C. Lechte, M. Bernert, G. D. Conway, S. J. Freethy, C. Honoré, J. R. Pinzón, and U. Stroth, Plasma Physics and Controlled Fusion 59, 054009 (2017).

[28] M. Willensdorfer, S. Denk, E. Strumberger, W. Suttrop, B. Vanovac, D. Brida, M. Cavedon, I. Classen, M. Dunne, S. Fietz, R. Fischer, A. Kirk, F. Laggner, Y. Liu, T. Odstrčil, D. Ryan, E. Viezzer, H. Zohm, and I. C. Luhmann, Plasma Physics and Controlled Fusion 58, 114004 (2016).

[29] H. Murmann, S. Götsch, H. Röhr, H. Salzmann, and K. Steuer, Review of Scientific Instruments 63, 4941
(1992).

[30] B. Kurzan and H. D. Murmann, Review of Scientific Instruments 82, 103501 (2011).

[31] E. Viezzer, T. Pütterich, R. Dux, and R. McDermott, Review of Scientific Instruments 83, 103501 (2012).

[32] R. Budny, M. Bell, H. Biglari, M. Bitter, C. Bush, C. Cheng, E. Fredrickson, B. Grek, K. Hill, H. Hsuan, A. Janos, D. Jassby, D. Johnson, L. Johnson, B. LeBlanc, D. McCune, D. Mikkelsen, H. Park, A. Ramsey, S. Sabbagh, S. Scott, J. Schivell, J. Strachan, B. Stratton, E. Synakowski, G. Taylor, M. Zarnstorff, and S. Zweben, Nuclear Fusion 32, 429 (1992).

[33] S. Sattler and H. Hartfuss, Plasma Physics and Controlled Fusion 35, 1285 (1993).

[34] G. Cima, Il Nuovo Cimento D 16, 359 (1994).

[35] C. Watts, R. Gandy, T. Rempel, and G. Cima, Review of Scientific Instruments 66, 451 (1995).

[36] C. Watts, Fusion Science and Technology 52, 176 (2007).

[37] S. L. Marple, Digital spectral analysis: with applications (Prentice-Hall, Inc., 1986).

[38] M. Häse, M. Hirsch, and H. Hartfuss, Review of Scientific Instruments 70, 1014 (1999).

[39] A. White, W. Peebles, T. Rhodes, C. Holland, G. Wang, L. Schmitz, T. Carter, J. Hillesheim, E. Doyle, L. Zeng, G. McKee, G. Staebler, R. Waltz, J. DeBoo, C. Petty, and K. Burrell, Physics of Plasmas 17, 056103 (2010).

[40] J. Hillesheim, W. Peebles, T. Rhodes, L. Schmitz, A. White, and T. Carter, Review of Scientific Instruments 81, 10D907 (2010).

[41] D. Smith, E. Powers, and G. Caldwell, IEEE Transactions on Plasma Science 2, 261 (1974).

[42] J. Hillesheim, J. DeBoo, W. Peebles, T. Carter, G. Wang, T. Rhodes, L. Schmitz, G. McKee, Z. Yan, G. Staebler, K. Burrell, E. Doyle, C. Holland, C. Petty, S. Smith, A. White, and L. Zeng, Physical Review Letters 110, 045003 (2013).

[43] J. Hillesheim, J. . DeBoo, W. Peebles, T. Carter, G. Wang, T. Rhodes, L. Schmitz, G. McKee, Z. Yan, G. Staebler, K. Burrell, E. Doyle, C. Holland, C. Petty, S. Smith, A. White, and L. Zeng, Physics of Plasmas 20, 056115 (2013).

[44] D. Prisiazhniuk, A. Krämer-Flecken, G. D. Conway, T. Happel, A. Lebschy, P. Manz, V. Nikolaeva, and U. Stroth, Plasma Physics and Controlled Fusion 59, 025013 (2017).

[45] R. Bravenec and A. Wootton, Review of Scientific Instruments 66, 802 (1995).

[46] M. Bornatici, R. Cano, O. De Barbieri, and F. Engelmann, Nuclear Fusion 23, 1153 (1983).

[47] S. Denk, R. Fischer, O. Maj, E. Poli, J. Stober, U. Stroth, B. Vanovac, W. Suttrop, and M. Willensdorfer, EPJ Web of Conferences 147, 02002 (2017).

[48] T. Eibert, E. Kilic, C. Lopez, R. Mauermayer, O. Neitz, and G. Schnattinger, Progress In Electromagnetics Research 151, 127 (2015).

[49] P. F. Goldsmith, Quasioptical Systems: Gaussian Beam Quasioptical Propogation and Applications (Wiley-IEEE Press, 1997).

[50] P. . Terry, M. Greenwald, J.-N. Leboeuf, G. McKee, D. Mikkelsen, W. Nevins, D. Newman, and D. Stotler, Physics of Plasmas 15, 062503 (2008).

[51] P. Ricci, C. Theiler, A. Fasoli, I. Furno, K. Gustafson, D. Iraji, and J. Loizu, Physics of Plasmas 18, 032109 (2011). 
[52] C. Holland, Physics of Plasmas 23, 060901 (2016). 